\title{
Valutazione pilota di un sistema automatico per lo screening anticorpale della toxoplasmosi
}

\author{
Pietro Casella', Annibale Raglio \\ ' Servizio di Medicina e Laboratorio, Azienda Ospedaliera "Ospedale Civile"di Vimercate: Presidio di Vimercate \\ 2 Servizio di Microbiologia e Virologia Azienda Ospedaliera "Ospedali Riuniti di Bergamo"
}

Key words: Toxoplasmosis, Toxoplasma gondii

A new automatic system for Toxoplasma antibodies screening: preliminary evaluation

\section{Summary}

The aim of this study was the comparison of a fully automated random access system IMMULITE 2000 (DPC) with a semiautomated system VIDAS (bioMérieux) for the first level detection of Toxoplasma-specific lgG and IgM antibodies. In total, 295 samples were examined for Toxoplasma- specific lgG. The comparison between the VIDAS IgG assay and IMMULITE IgG assay showed the same result for 290 samples (98.3\%). Particularly, 220 samples were negative, 70 positive and 4 samples were positive with IMMULITE and equivocal with VIDAS. Only one sample showed a discrepant result: positive with IMMULITE and negative with VIDAS.

A total of 124 samples were tested for Toxoplasma-specific IgM and 33 were positive or equivocal with VIDAS $\lg M$ assay and 30 of these were also tested for Toxoplasma-specific IgG avidity.

The comparison between the VIDAS IgM assay and IMMULITE IgM assay showed the following results: 23 samples were positive in the two systems, 4 samples were equivocal with the VIDAS IgM assay but positive in IMMULITE IgM assay, 6 samples were positive or equivocal in the VIDAS IgM assay but negative in IMMULITE IgM assay. The study of the 6 discrepant samples showed: 2 samples with the presence of natural IgM, 3 samples with a high IgG avidity index and I with an intermediate IgG avidity index. IgM was not detected with both assays in any of the other samples. The IMMULITE system seems to be a valuable system for the first level diagnosis of Toxoplasma infection.

Scopo del lavoro è stata la valutazione del sistema automatico ad accesso random IMMULITE 2000 (DPC) rispetto al sistema semiautomatico VIDAS (bioMérieux) per lo screening anticorpale di primo livello della toxoplasmosi. Lo studio è stato condotto in singolo su sieri ad attività nota per VIDAS provenenti da una popolazione di soggetti immunocompetenti. Per la determinazione delle IgG sono stati saggiati 295 sieri. La concordanza tra i due metodi è stata del 98.3\% (290/295) di cui 70 positivi, 22 I negativi, 4 dubbi per VIDAS e positivi per IMMULITE. Un siero risultava discordante (positivo per IMMULITE e negativo per VIDAS). Per la determinazione delle IgM sono stati saggiati 124 sieri di cui 33 con IgM positive o dubbi per VIDAS (30 dei quali ad avidità nota). Di questi 23 sieri erano positivi in entrambi i metodi, 6 negativi per IgM secondo IMMULITE e positivi o dubbi per VIDAS (3 con avidità elevata, I con avidità intermedia e 2 IgM naturali), 4 positivi per IMMULITE e dubbi per VIDAS. Nel rimanente dei sieri le IgM erano assenti in entrambi i metodi. La nostra esperienza con il sistema Immulite 2000 si è dimostrata positiva.

\section{INTRODUZIONE}

Toxoplasma gondii è un protozoo appartenente all'ordine dei coccidi a diffusione mondiale. L'infezione colpisce molti vertebrati, uomo compreso, dove rappresenta una delle più frequenti infezioni parassitarie (1). La via di contagio più comune nell'uomo avviene soprattutto attraverso l'ingestione di carne cruda o poco cotta, meno frequentemente dall'ingestione accidentale di oocisti eliminate dal gatto (ospite definitivo), in utero, per trasfusione e per trapianto d'organo (1). Nei soggetti immunocompetenti l'infezione acuta è di solito asintomatica e anche nei casi sintomatici la guarigione di solito è spontanea, tuttavia l'infezione primaria in gravidanza, per il rischio di trasmissione del parassita al prodotto del con- cepimento, rappresenta una delle maggiori sfide per la sierodiagnosi parassitologica (4). La diagnosi di toxoplasmosi viene posta per lo più su base sierologica avvalendosi di svariate tecniche. Data la diffusione dell'infezione la toxoplasmosi rappresenta la malattia parassitaria per la quale a livello mondiale viene fatto il maggior numero di esami sierologici. Nella maggior parte dei laboratori l'approccio diagnostico più frequente riguarda la definizione dello status immunologico in pazienti gravide o prossime alla gravidanza e la diagnosi di infezione acuta in soggetti immunocompetenti (7). L'approccio metodologico più diffuso per un test di primo livello comprende la ricerca di IgG e IgM con un metodo sensibile (9). L'elevato numero di determinazioni che di solito 
vengono saggiate in laboratorio ha spinto le società produttrici di diagnostici a produrre metodi in completa automazione. Scopo del lavoro è la valutazione di un metodo automatico ad accesso random su provetta primaria che permette la ricerca di $\operatorname{IgG}$ e IgM con tecnologia a cattura (IMMULITE 2000, DPC) per la diagnosi di primo livello su un campione di sieri provenienti da una popolazione costituita per lo più da donne gravide precedentemente saggiati con il sistema da noi in uso (VIDAS bio Merieux).

\section{MATERIALI E METODI Campioni}

L'indagine è stata condotta utilizzando 295 campioni di siero. Di tutti questi sono state dosate le IgG con entrambi i metodi. La determinazione delle IgM è stata fatta in 124 di questi campioni. Fra questi 33 erano sieri congelati, positivi o dubbi per IgM con tecnologia VIDAS appartenenti a 29 femmine in gravidanza e 4 maschi.

\section{IgG e IgM VIDAS}

Il principio del metodo associa la tecnica immunoenzimatica a sandwich ( $\operatorname{IgG})$ o a cattura $(\operatorname{IgM})$ ad una rilevazione in fluorescenza (ELFA). Con esclusione della fase di pipettamento le altre fasi del test sono realizzate automaticamente. Per le $\mathrm{IgG}$ i risultati vengono espressi in U.I. $/ \mathrm{ml}$. Titoli $>/=8$ sono da considerare positivi, $<4$ negativi, tra 4 e 7 dubbi.

Le IgM sono rilevate con tecnologia a cattura. Nel nostro lavoro VIDAS è stato utilizzato come metodo di riferimento.

\section{IgG e IgM IMMULITE}

IMMULITE 2000 è un sistema ad alta produttività recentemente sviluppato nel settore infettivologico, in particolare per il complesso ToRCH. Il sistema completamente automatico ad accesso random da provetta primaria, utilizza tecnologia in chemiluminescenza ed è corredato di software per la gestione del controllo di qualità interno in tempo reale. La determinazione delle IgG è realizzata in formato sandwich mentre le IgM utilizzano tecnologia a cattura. Per IgG i risultati sono espressi in U.I./ml Valori $>/=8$ sono da considerare positivi, $<6.5$ negativi, tra 6.5 e $<8$ indeterminati. Le IgM sono rilevate con tecnologia a cattura.

\section{Avidità}

Il test di avidità è stato condotto con la strumentazione VIDAS. L'avidità delle IgG prodotte dopo il primo impatto antigenico nell'infezione primaria è bassa e aumenta nelle settimane e mesi successivi in seguito alla selezione delle cellule B, ne consegue che le IgG derivanti da infezione pregressa o da risposta secondaria hanno avidità elevata (4). Il principio utilizza una cartuccia doppia, composta da una di riferimento e una cartuccia test dove è presente l'agente dissociante. Nella cartuccia test in presenza dell'agente dissociante $\mathrm{i}$ legami antigene anticorpo vengono alterati, pertanto IgG non mature per i determinanti antigenici, indicative di infezione recente o in atto, vengono significativamente dissociate dall'antigene presente sulla fase solida, mentre le IgG mature restano agganciate alla fase solida. Il rapporto tra le concentrazioni di anticorpi tra la cartuccia test e la cartuccia di riferimento è indicativo dell'avidità degli anticorpi presenti nel campione. Un rapporto $>/=0.300$ è indice di forte avidità (infezione primaria contratta da più di 4 mesi), mentre un rapporto $<$ a 0.200 è suggestivo di infezione recente.

\section{Controllo dei campioni discordanti}

$\mathrm{Su}$ alcuni campioni discordanti è stata fatta una verifica con il sistema completamente automatizzato LIAISON (Dia Sorin). Sono state determinate $\mathrm{IgG}, \mathrm{IgM}$ e avidità.

\section{Controlli di qualità}

In ogni seduta analitica sono stati inseriti controlli di qualità interni sia per IgG che per IgM.

\section{RISULTATI}

\section{Determinazione di IgG}

I risultati della sperimentazione sono riportati in tabella 1. Dei 295 sieri saggiati 70 erano positivi per VIDAS che IMMULITE, 221 Negativi e 5 discordanti. La concordanza tra i 2 metodi per lo screening delle IgG è stata del 98.3\% (290/295). Tra i 5 discordanti 4 erano dubbi per VIDAS e positivi IMMULITE 5 vs 9,4 vs 14,7 vs 10,7 vs 11 U.I./ml. Infine un campione di siero era negativo per VIDAS e positivo IMMULITE 0 vs 12 U.I./ml

\section{DETERMINAZIONE DI IGM}

La determinazione delle IgM è stata condotta con 124 campioni di siero, di questi 33 erano a reattività nota (IgM positive o dubbie con VIDAS). Per 30 di questi campioni era stato eseguito anche il test di avidità. 29 sieri appartenevano a donne gravide e 4 a uomini.

Per il dosaggio delle IgM 91 sieri erano negativi per entrambi i metodi. I risultati del pannello di sieri noti sono riportati in dettaglio in tabella 2.

Del pannello dei sieri 33 sieri noti, 22 campioni erano concordanti per entrambi i metodi, tra questi 10 con infezione recente $\mathrm{o}$ in atto (avidità $<\mathrm{di}$ $0.125)$. In 4 campioni le IgM erano dubbie per 
VIDAS e positive per IMMULITE. (avidità > 0.300 ).

Gli ultimi 6 campioni presentavano delle discordanze (ultimi sieri in tabella 2). Di questi, 5 campioni erano positivi per VIDAS e negativi per IMMULITE (CM, AM, EH, GF, e CX). 2 di questi con IgM positive e IgG assenti erano di donne gravide (CM e AM). Presumibilmente si trattava di IgM naturali, infatti nel follow up durante la gravidanza non sono mai comparse $\mathrm{IgG}$ e dopo il parto anche VIDAS non rilevava le IgM precedentemente segnalate. 2 campioni anch'essi di donne gravide (EH e $\mathrm{CX}$ ) con $\mathrm{IgG}$ rispettivamente di 80 e 199 e IgM positive per VIDAS e negative per IMMULITE con avidità superiore $\mathrm{a}$ 0.300 . Di queste due pazienti non è stato possibile ottenere altre informazioni. Infine un siero di un maschio (GF) ricoverato in cardiologia saggiato per sospetta toxoplasmosi con $\mathrm{IgG}$ di 12 U.I./ml e IgM presenti con VIDAS e avidità $>0.300$.

L'ultimo caso discordante (BR) si trattava di una donna gravida con IgG 154 U.I./ml e IgM dubbie per VIDAS e negative per IMMULITE, con avidità di 0.221 (zona grigia). La storia di questa paziente, dal 17/05/2002 al 10/10/2003, è rappresentata da un'alternanza di deboli positività per IgM per la rosolia poi scomparse quindi da un comparsa di IgM dubbie per toxoplasmosi ma con avidità intermedia $(0.221)$ quando la paziente è diventata gravida $(14 / 02 / 2003)$, fino alla scomparsa delle IgM (10/10/2003). Il livello delle IgG è sempre rimasto pressoché costante dal primo dosaggio.

La verifica di questi 6 campioni discordanti con il sistema LIAISON ha confermato quanto rilevato con VIDAS In tutti i sieri erano presenti IgM (anche le 2 naturali) e tutti, con esclusione naturalmente di questi ultimi mostravano avidità elevata.

\section{DISCUSSIONE}

La sierodiagnosi per la toxoplasmosi è un evento estremamente complesso, anche per un approccio sierologico di primo livello, per questo definiamo il lavoro come esperienza preliminare, infatti data la complessità del problema siamo consapevoli che ben altri numeri dovrebbero comprendere la casistica da noi esaminata.

Il sistema VIDAS utilizzato come riferimento rappresenta una garanzia di provata affidabilità in quanto i test prodotti da bioMérieux rappresentano validi presidi diagnostici per questa sierodiagnosi (6). La valutazione dello status immunologico per le donne gravide o in previsione di un imminente gravidanza costituisce un pesante carico di lavoro che gravita soprattutto sui laboratori non specialistici (7). La prevalenza di sieropositività presso questa popolazione è bassa e mediamente intorno al $29 \%$ delle donne in età fertile di età compresa tra i 21 e i 40 anni (10), pertanto tutte le gravide sieronegative effettuano controlli con cadenza mensile fino alla fine della gestazione. In Italia lo schema dei controlli, a differenza di quello proposto dal CDC, che prevede la sola determinazione delle IgG per le gravide sieronegative (3), si basa sul dosaggio di IgG e IgM, e l'impiego di un sistema completamente automatico da provetta primaria rappresenta la scelta ottimale poiché riduce in modo significativo gli errori della fase preanalitica.

Un test automatico di screening per IgG e IgM deve essere caratterizzato da performance imprescindibili sulla specificità per le IgG e sulla sensibilità per le IgM, in particolare per queste ultime è irrinunciabile la tecnologia a cattura (4). La buona concordanza con VIDAS per le IgG ha posto il sistema IMMULITE su un piano qualitativo di notevole interesse. Nella nostra esperienza abbiamo riscontrato 4 sieri considerati dubbi per VIDAS e positivi per IMMULITE e un siero con 12 U.I./ml per IMMULITE e negativo per VIDAS; pertanto fino a definitivi riscontri riteniamo prudentemente che, donne gravide con bassi livelli di IgG (fino a $15 \mathrm{U} . \mathrm{I} / \mathrm{ml}$ ) non andrebbero considerate protette ma invitate a controlli periodici mensili e a osservare tutte quelle norme igienico comportamentali raccomandate per i soggetti non protetti (7).

Sarebbe anche opportuno affiancare per un certo periodo ad un nuovo sistema un test di secondo livello per la ricerca di IgG in quanto è stato segnalato che alcuni test ELISA per IgG corrono il rischio di segnalare false positività (7). Relativamente alle IgM IMMULITE si è dimostrato sicuramente specifico, sono stati rilevati come negativi i due casi di IgM naturali evidenziati da VIDAS e da LIAISON, anche la sensibilità ci è sembrata buona, infatti i 10 casi di presunta infezione in atto sono stati rilevati con IMMULITE, presunta perché mentre un'avidità elevata esclude l'infezione recente una bassa avidità non necessariamente la include (4).

Tutti gli altri casi con IgM positive o indeterminate per VIDAS con infezione antecedente ai quattro mesi (elevata avidità) sono stati correttamente rilevati.

Per i due casi di donne gravide e il soggetto maschio con IgM positive con VIDAS e negative con IMMULITE ma con avidità $>0,300$ la non rilevazione da parte di IMMULITE non ci è sembrata di particolare gravità in quanto l'infezione era presumibilmente antecedente ad almeno 4 mesi (l'elevata avidità è stata dimostrata anche 
con LIAISON).

Circa la donna gravida (BR ultima nella tabella 2) con quella situazione sierologica piuttosto confusa possiamo ipotizzare che la stabilità delle $\mathrm{IgG}$ per 12 mesi sembra escludere un infezione in atto. Va inoltre rilevato che mentre per VIDAS l'avidità si collocava in zona grigia per LIAISON l'avidità era elevata.

Va sottolineato che il sistema LIAISON da noi usato per verificare i sieri con IgM discordanti si dimostrato affidabile in un lavoro di recente pubblicazione (11). Nella nostra casistica IMMULITE è stato in grado di rilevare tutti i soggetti con infezione recente.

Questi dati dimostrano che l'utilizzo di un gestionale che consente la rintracciabilità delle storie dei pazienti insieme all'imprescindibile apporto del test di avidità rappresentano requisiti indispensabili per la conferma e la databilità dell'esordio delle infezioni.

Lo sviluppo del test di avidità ha rivoluzionato la diagnosi sierologica delle infezioni da Toxoplasma gondii, infatti la misura dell'avidità delle IgG ha dimostrato di essere di estrema utilità abbinata a altri test convenzionali come il dosaggio di IgG e IgM con saggi EIA $(4,8)$.

L'infezione acuta può essere diagnosticata con un singolo campione di siero e il test si è rivelato particolarmente utile nel datare l'infezione nelle popolazione immunocompetente data la frequente asintomaticità dell'infezione, la linfoadenopatia compare dal 3 al 7\% dei soggetti immunocompetenti $(2,5)$, e per il perdurare di IgM specifiche soprattutto in gravidanza (4).

Tabella I. Confronto IgG VIDAS verso IgG IMMULITE

\begin{tabular}{lr} 
& N. CAMPIONI \\
\hline Negativi VIDAS e IMMULITE & 22 I \\
\hline Positivi VIDAS e IMMULITE & 70 \\
\hline Indeterminati VIDAS, Positivi IMMULITE & 4 \\
\hline Positivi IMMULITE, Negativi VIDAS & I \\
\hline
\end{tabular}

TOTALE 295

Tabella 2. Pannello dei sieri reattivi per $\lg M$

\begin{tabular}{|c|c|c|c|c|c|c|}
\hline PAZIENTE & $\begin{array}{c}\text { IgG } \\
\text { VIDAS }\end{array}$ & $\begin{array}{c}\text { IgG } \\
\text { IMMULITE }\end{array}$ & $\begin{array}{c}\text { IgM } \\
\text { VIDAS }\end{array}$ & $\begin{array}{c}\text { IgM } \\
\text { IMMULITE }\end{array}$ & Avidità & Note \\
\hline TA & 84 & 106 & PRESENTI & PRESENTI & 0,557 & \\
\hline PP & $>300$ & $>250$ & PRESENTI & PRESENTI & - & \\
\hline GE & 113 & $>250$ & PRESENTI & PRESENTI & 0,068 & \\
\hline PX & 300 & $>250$ & PRESENTI & PRESENTI & - & \\
\hline CM & 298 & $>250$ & PRESENTI & PRESENTI & 0,103 & \\
\hline CS & 147 & $>250$ & PRESENTI & PRESENTI & 0,109 & \\
\hline $\mathrm{CL}$ & 280 & $>250$ & PRESENTI & PRESENTI & 0,287 & \\
\hline $\mathrm{NC}$ & & & PRESENTI & PRESENTI & - & \\
\hline$C M$ & 162 & 163 & PRESENTI & PRESENTI & 0,428 & \\
\hline$A X$ & 69 & 85 & PRESENTI & PRESENTI & 0,315 & \\
\hline SL & 32 & 97 & PRESENTI & PRESENTI & 0,046 & \\
\hline$S L$ & 129 & $>250$ & PRESENTI & PRESENTI & 0,074 & \\
\hline RF & 6 & 12 & PRESENTI & PRESENTI & 0,056 & \\
\hline AY & $|4|$ & $>250$ & PRESENTI & PRESENTI & 0,457 & \\
\hline GX & 12 & 22 & PRESENTI & PRESENTI & 0,072 & \\
\hline DC & $>300$ & $>250$ & PRESENTI & PRESENTI & 0,457 & \\
\hline GX & 24 & 62 & PRESENTI & PRESENTI & 0,094 & \\
\hline $\mathrm{BX}$ & 50 & 63 & PRESENTI & PRESENTI & 0,351 & \\
\hline QX & 176 & 200 & PRESENTI & PRESENTI & 0,552 & \\
\hline FX & 62 & 150 & PRESENTI & PRESENTI & 0,018 & \\
\hline $\mathrm{BI}$ & 37 & 55 & PRESENTI & PRESENTI & 0,05 & \\
\hline AY & $>300$ & $>250$ & PRESENTI & PRESENTI & - & \\
\hline SF & $>300$ & $>250$ & PRESENTI & PRESENTI & 0,482 & \\
\hline CS & 129 & $>250$ & DUBBIO & PRESENTI & 0,331 & \\
\hline DM & $>300$ & $>250$ & DUBBIO & PRESENTI & 0,653 & \\
\hline $\mathrm{NC}$ & $>300$ & $>250$ & DUBBIO & PRESENTI & 0,514 & \\
\hline GW & 73 & 133 & DUBBIO & PRESENTI & 0,423 & \\
\hline CM & 0 & $<5$ & PRESENTI & ASSENTI & - & IgM NATURALI \\
\hline AM & 0 & $<5$ & PRESENTI & ASSENTI & - & IgM NATURALI \\
\hline $\mathrm{EH}$ & 199 & $>250$ & PRESENTI & ASSENTI & 0,438 & \\
\hline GF & 13 & 24 & PRESENTI & ASSENTI & 0,415 & \\
\hline$C X$ & 80 & 153 & PRESENTI & ASSENTI & 0,365 & \\
\hline BR & 144 & $>250$ & DUBBIO & ASSENTI & 0,221 & \\
\hline
\end{tabular}


Per la determinazione dell'avidità delle IgG sono disponibili commercialmente diversi kit, in particolare quello da noi usato si è dimostrato affidabile per donne in gravidanza immunocompetenti (6). Per il monitoraggio della toxoplasmosi nelle gravide è fondamentale un approccio primario con test di IgG e di IgM a elevata sensibilità supportato da test di avidità per confermare o escludere la possibile infezione recente (4).

È fondamentale che in tutti i laboratori vengano conservati almeno tutti i campioni reattivi o dubbi per IgM e che, in prima persona, i laboratoristi si attivino per affidare $i$ casi poco chiari o rilevanti come: toxoplasmosi congenita, oculare $\mathrm{e}$ in pazienti immunosoppressi a un centro di riferimento o ad un laboratorio di livello superiore.

Sarà compito del centro di livello superiore scegliere le opportune strategie diagnostiche: sierologiche o di biologia molecolare per la gestione di questi casi complessi.

\section{CONCLUSIONI}

Il sistema IMMULITE 2000 si è dimostrato promettente per la diagnosi di primo livello per la ricerca di IgG e IgM in una popolazione di soggetti immunocompetenti comprendenti donne in gravidanza. Data la complessità e la rilevanza clinica dell' argomento riteniamo comunque che altri studi andrebbero condotti per confermare quanto emerso dalla nostra esperienza pilota.

\section{BIBLIOGRAFIA}

1. Garcia LS. Toxoplasma gondii in: Diagnostic Medical Parasitology. 132-142 Fourth Ed 2001 ASM Press Washington, D.C.

2. Gard S, Magnusson JH. A glandular form of toxoplasmosis in connection with pregnancy. Acta Med Scand 1951; 161: 59-64.

3. http://www.dpd.cdc.gov/dpdx/HTML/Toxoplasmosis.htm

4. Lappalainen M, Hedman K. Serodiagnosis of Toxoplasmosis. The Impact of Measurement of IgG Avidity. Ann Ist Super Sanità 2004; 40(1): 81-8.

5. McCabe RE, Brooks RG, Dorfman RF, Remington JS. Clinical Spectrum in 107 Cases of Toxoplasmic Lynphadenopathy. Rev Infect Dis 1987; 9: 754-74.

6. Pelloux, Brun E, Vernet $\mathrm{G}$, Marcillant S, Jolivet Guergour D, Fricker-Hidalgo A, Ambroise-Thomas P. Determination of anti-Toxoplasma gondii Immunoglobulin G Avidity: Adaptation to the Vidas System (bioMérieux). Diagn Microbiol Infect Dis 1998; 32: 69-73.

7. Raglio A, Marty P, Cutrupi V, Rigoli E, Rescaldani R. Criteri di Diagnosi Siero-immunologica nelle Infezioni da Toxoplasma gondii. Microbiologia Medica 1998; 13; 50-59.

8. Rescaldani R, Vigorè L, Vezzo R, Bramati S, Filtri G, Bonaffini A, Casella P. Toxoplasmosi Sintomatica e Test di Avidità delle IgG. Microbiologia Medica 1992; 4 (7): 96-8.

9. Roberts A, Hedman K, Luyasu V, et al. Multicenter Evaluation of Strategies for Serodiagnosis of Primary Infection with Toxoplasma gondii. Eur J Clin Microbiol Infect Dis 2001; 20: 467-74.

10. Vezzo R, Vigorè L, Goglio A, et al. Sieroprevalenza per Anticorpi Anti-Toxoplasma gondii in 14186 soggetti. Microbiologia Medica 2001 V16, 2, M099

11. Petersen E, Borobio MV, Guy E, et al. European multicenter study of the Liaison automated diagnostic system for determination of Toxoplasma gondii-specific immunoglobulin $\mathrm{G}$ ( $\mathrm{IgG})$ and $\operatorname{IgM}$ and the $\operatorname{IgG}$ Avidity index. J Clin Microbiol 2005; 43 (4), 1570-4.
Pietro Casella

Via Torino 22, 20040 Usmate (MI)

Tel. 039 6654591, Fax.039 66544772

E-mail: casella.pietro@aovimercate.org 